\title{
An Application of Distance and Similarity Measure of Fuzzy Soft Set in Decision Making and Medical Diagnosis
}

\author{
Dr. N. Sarala ${ }^{1}$, B. Suganya ${ }^{2}$ \\ ${ }^{1}$ Department of Mathematics, A.D.M College for women, Nagapattinam (India) \\ ${ }^{2}$ Department of Mathematics, Bharathidasan University Constituent College of arts and science, Nagapattinam.(India)
}

\begin{abstract}
Similarity Measure is a very important problem in Fuzzy Soft Set theory. In this paper, we have applied the notion of Distance and Similarity measures between two fuzzy soft set. First we discussed Decision making problem using fuzzy soft set based real life problems. Finally the similarity measures of normalized Hamming distance and normalized Euclidean distance have been applied to the problem in medical diagnosis.
\end{abstract}

Keywords: Soft set, Fuzzy soft set, Distance Measure, Similarity Measure, normalized Hamming distance, normalized Euclidean distance

\section{Introduction}

In many complicated problems arising in the fields of engineering, social science, economics, medical science etc involving uncertainties, classical methods are found to be inadequate in recent times. Molodtsov initiated soft set theory [8] as a new mathematical tool for dealing with uncertainties. In recent times, researchers have contributed a lot towards fuzzification of soft set theory.

Maji et al [6] introduced the concept of fuzzy soft set and some properties regarding fuzzy soft union, intersection, complement of a fuzzy soft set etc. Similarity measure have extensive application in pattern recognition, region extraction, coding theory, image processing and in many other areas. Similarity measure is a very important issue in fuzzy soft sets. In recent years, similarity measures between fuzzy soft sets have been studied from different aspects and applied in various field.

In this paper, we first present the basic definitions of soft sets, fuzzy sets, fuzzy soft sets that are useful for subsequent discussions. We then define distances and similarity measures between two fuzzy soft set (FSS) sets. By using the similarity we construct a decision making method. We finally give an application.

Next we will study some new similarity measures of fuzzy soft sets based on the normalized Hamming distance, the normalized Euclidean distance, an example is given to illustrate the application of these new similarity measures of fuzzy soft sets in medical diagnosis.

Throughout this work, $U$ refers to the initial universe, $P(U)$ is the power set of $U, E$ is a set of parameters and $A \subseteq E . S(U)$ denotes the set of all soft sets over $U$.

\section{Preliminaries}

In this section, we first recall the basic definitions related to fuzzy soft sets which would be used in the sequel.

Definition 2.1. Soft set [8]

Suppose that $U$ is an initial universe set and $E$ is a set of parameters, let $\mathrm{P}(\mathrm{U})$ denotes the power set of $\mathrm{U}$. A pair $(F, E)$ is called a soft set over $U$ where $F$ is a mapping given by $\mathrm{F}: \mathrm{E} \rightarrow \mathrm{P}(\mathrm{U})$.

Clearly, a soft set is a mapping from parameters to $\mathrm{P}(\mathrm{U})$, and it is not a set, but a parameterized family of subsets of the Universe.

\section{Definition 2.2. Fuzzy soft set [6]}

Let $U$ be an initial Universe set and $E$ be the set of parameters. Let $A \subset E$. A pair $(F, A)$ is called fuzzy soft set over $U$ where $F$ is a mapping given by $F$ : $A \rightarrow I^{U}$, where $I^{U}$ denotes the collection of all fuzzy subsets of $U$.

\section{Example 2.3}

Let $\mathrm{U}$ be the set of five vehicle under consideration and $\mathrm{E}=\left\{\mathrm{e}_{1}\right.$ (costly), $\mathrm{e}_{2}$ (fuel) $,\left(\mathrm{e}_{3}\right.$ beautiful $),\left(\mathrm{e}_{4}\right.$ modern technology $\left.)\right\}$ be the set of parameters and $\mathrm{A}=\left\{\mathrm{e}_{1}, \mathrm{e}_{2}, \mathrm{e}_{3}\right\} \subseteq \mathrm{E}$. then

$(\mathrm{F}, \mathrm{A})=\left\{\mathrm{F}\left(\mathrm{e}_{1}\right)=\left\{\mathrm{c}_{1 /} 0.1, \mathrm{c}_{2} / 0.2, \mathrm{c}_{3} / 0.7, \mathrm{c}_{4} / 0.6\right\}\right.$

$\left\{\mathrm{F}\left(\mathrm{e}_{2}\right)=\left\{\mathrm{c}_{1 /} 0.7, \mathrm{c}_{2} / 0.5, \mathrm{c}_{3} / 0.2, \mathrm{c}_{4} / 0.4\right\}\right.$

$\left\{\mathrm{F}\left(\mathrm{e}_{3}\right)=\left\{\mathrm{c}_{1 /} 0.2, \mathrm{c}_{2} / 0.1, \mathrm{c}_{3} / 0.6, \mathrm{c}_{4} / 0.2\right\}\right.$

Definition 2.4. Fuzzy soft class [9]

Let $U$ be an initial Universe set and $\mathrm{E}$ be the set of attributes. Then the pair (U,E) denotes the collection of all fuzzy soft sets on $U$ with attributes from $E$ and is called a fuzzy soft class.

Definition 2.5. Union of fuzzy soft sets [10]

Union of two fuzzy soft sets (F,A) and $(\mathrm{G}, \mathrm{B})$ in a soft class $(\mathrm{U}, \mathrm{E})$ is a fuzzy soft set $(\mathrm{H}, \mathrm{C})$ where $C=A \cup B$ and 


\section{International Journal of Science and Research (IJSR)}

ISSN (Online): 2319-7064

Index Copernicus Value (2013): 6.14 | Impact Factor (2014): 5.611

\section{$H(e)= \begin{cases}F(e) & \text { if } e \in A-B \forall e \in C, \\ G(e) & \text { if } e \in B-A \\ F(e) \cup G(e) & \text { if } e \in A \cap B\end{cases}$}

and is written as $(\mathrm{F}, \mathrm{A}) \cup(\mathrm{G}, \mathrm{B})=(\mathrm{H}, \mathrm{C})$

Definition 2.6. Intersection of fuzzy soft sets [2]

Intersection of two fuzzy soft sets $(\mathrm{F}, \mathrm{A})$ and $(\mathrm{G}, \mathrm{B})$ in a soft

class (U,E) is a fuzzy soft set $(H, C)$ where $\mathrm{C}=\mathrm{A} \cap \mathrm{B}$ and

$$
\forall \mathrm{e} \in \mathrm{C}, \mathrm{H}(\mathrm{e})=\mathrm{F}(\mathrm{e}) \text { or } \mathrm{G}(\mathrm{e})
$$

and is written as

$$
(\mathrm{F}, \mathrm{A}) \cap(\mathrm{G}, \mathrm{B})=(\mathrm{H}, \mathrm{C})
$$

\section{Definition 2.7. Complement of a fuzzy soft set [10]}

The complement of a fuzzy soft set (F,A) is denoted by $(\mathrm{F}, \mathrm{A})^{\mathrm{c}}$ and is defined by $(\mathrm{F}, \mathrm{A})^{\mathrm{c}}=\left(\mathrm{F}^{\mathrm{c}}, \mathrm{A}\right)$, where $\mathrm{F}^{\mathrm{c}}: \mathrm{A} \rightarrow \mathrm{P}(\mathrm{U})$ is a mapping given by $\mathrm{F}^{\mathrm{c}}(\mathrm{e})=(\mathrm{F}(\mathrm{e}))^{\mathrm{c}}, \quad \forall \mathrm{e} \in \mathrm{A}$.

\section{Distance between Fuzzy Soft Set}

Distance and similarity measures have attracted a lot of attentions in the last few decades due to the fact that they can be applied to many areas such as pattern recognition, cluster analysis, approximate reasoning, image processing, medical diagnosis and decision making. In this section, we analyses about the axiomatic definitions of the distance measures as follows.

\section{Definition 3.1.}

Let $(\mathrm{F}, \mathrm{A}),(\mathrm{G}, \mathrm{B})$ and $(\mathrm{H}, \mathrm{C})$ be fuzzy soft sets in a fuzzy soft space $(X, E)$ and $d: X \times X \rightarrow R^{+}$a mapping. Then

(1) $d$ is said to be quasi-metric if it satisfies

$\left(\mathrm{M}_{1}\right) \quad \mathrm{d}((\mathrm{F}, \mathrm{A}),(\mathrm{G}, \mathrm{B})) \geq 0$,

$\left(\mathrm{M}_{2}\right) \mathrm{d}((\mathrm{F}, \mathrm{A}),(\mathrm{G}, \mathrm{B}))=\mathrm{d}((\mathrm{G}, \mathrm{B}),(\mathrm{F}, \mathrm{A}))$,

(2) A quasi-metric $d$ is said to be semi-metric if

$\left(\mathrm{M}_{3}\right) \mathrm{d}((\mathrm{F}, \mathrm{A}),(\mathrm{G}, \mathrm{B}))+\mathrm{d}((\mathrm{G}, \mathrm{B}),(\mathrm{H}, \mathrm{C}))$

$$
\geq \mathrm{d}((\mathrm{F}, \mathrm{A}),(\mathrm{H}, \mathrm{C}))
$$

(3) A semi-metric $d$ is said to be pseudo metric if $\left.\left(\mathrm{M}_{4}\right)(\mathrm{F}, \mathrm{A})=(\mathrm{G}, \mathrm{B})\right) \Rightarrow \mathrm{d}((\mathrm{F}, \mathrm{A}),(\mathrm{G}, \mathrm{B}))=0$,

(4) A pseudo metric $d$ is said to be metric if

$\left(\mathrm{M}_{5}\right) \mathrm{d}((\mathrm{F}, \mathrm{A}),(\mathrm{G}, \mathrm{B}))=0 \Rightarrow(\mathrm{F}, \mathrm{A})=(\mathrm{G}, \mathrm{B})$.

\section{Definition 3.2}

For two fuzzy soft sets $(F, A)$ and $(G, B)$ in a fuzzy soft space $(\mathrm{X}, \mathrm{E})$, we define Cardinality semi-metric as: c $((\mathrm{F}, \mathrm{A}),(\mathrm{G}, \mathrm{B}))=\|\mathrm{A}\|-\|\mathrm{B}\| \mid+\sum\|\mathrm{F}(\mathrm{e})\|-\|\mathrm{G}(\mathrm{e})\|$

\section{Definition 3.3}

Let $(F, A)$ and $(\mathrm{G}, \mathrm{B})$ be two fuzzy soft sets over (U,E). Then distance measure between $(\mathrm{F}, \mathrm{A})$ and $(\mathrm{G}, \mathrm{B})$ is defined as $\mathrm{d}$ $((\mathrm{F}, \mathrm{A}),(\mathrm{G}, \mathrm{B}))$, which satisfies the following properties.

(D1) $0 \leq \mathrm{d}((\mathrm{F}, \mathrm{A}),(\mathrm{G}, \mathrm{B})) \leq 1$;

(D2) d ((F,A), (G,B)) $=0$, if $(\mathrm{F}, \mathrm{A})=(\mathrm{G}, \mathrm{B})$;

(D3) $\mathrm{d}((\mathrm{F}, \mathrm{A}),(\mathrm{G}, \mathrm{B}))=\mathrm{d}((\mathrm{G}, \mathrm{B}),(\mathrm{F}, \mathrm{A}))$;

(D4) Let $(H, C)$ be a fuzzy soft set,

if $(\mathrm{F}, \mathrm{A}) \subseteq(\mathrm{G}, \mathrm{B}) \subseteq(\mathrm{H}, \mathrm{C})$.

then $\mathrm{d}((\mathrm{F}, \mathrm{A}),(\mathrm{G}, \mathrm{B})) \leq \mathrm{d}((\mathrm{F}, \mathrm{A}),(\mathrm{H}, \mathrm{C}))$ and $\mathrm{d}((\mathrm{G}, \mathrm{B}),(\mathrm{H}, \mathrm{C}))$ $\leq \mathrm{d}((\mathrm{F}, \mathrm{A}),(\mathrm{H}, \mathrm{C}))$.

\section{Definition 3.4.}

For two fuzzy soft sets $(F, A)$ and $(G, B)$ in a fuzzy soft space $(\mathrm{X}, \mathrm{E})$, where $\mathrm{A}$ and $\mathrm{B}$ are not identically void, we define Euclidean distance as:

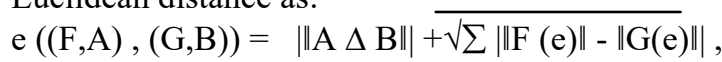

Various distance measures $d$ are present in literature. The most commonly employed distance measures are:

1. The Hamming distance

$\mathrm{d}_{\mathrm{H}}((\mathrm{F}, \mathrm{A}),(\mathrm{G}, \mathrm{B}))=\sum_{i=1^{*}}^{m} \cdot \sum_{j=1^{*}}^{n}\left|\mathrm{~F}\left(\mathrm{e}_{\mathrm{i}}\right)\left(\mathrm{x}_{\mathrm{j}}\right)-\mathrm{G}\left(\mathrm{e}_{\mathrm{i}}\right)\left(\mathrm{x}_{\mathrm{j}}\right)\right|$

2. The normalized Hamming distance

$$
\begin{array}{r}
\mathrm{d}_{\mathrm{nH}}((\mathrm{F}, \mathrm{A}),(\mathrm{G}, \mathrm{B}))=\frac{1}{m n} \sum_{i=1^{*}}^{m} \cdot \sum_{j=1^{*}}^{n} \mid \mathrm{F}\left(\mathrm{e}_{\mathrm{i}}\right)\left(\mathrm{x}_{\mathrm{j}}\right) \\
-\mathrm{G}\left(\mathrm{e}_{\mathrm{i}}\right)\left(\mathrm{x}_{\mathrm{j}}\right) \mid
\end{array}
$$

3. The Euclidean distance

$$
\begin{aligned}
\mathrm{d}_{\mathrm{E}}((\mathrm{F}, \mathrm{A}),(\mathrm{G}, \mathrm{B}))=\left\{\sum_{i=1}^{m}, \sum_{j=1}^{n},\right. & \mid \mathrm{F}\left(\mathrm{e}_{\mathrm{i}}\right)\left(\mathrm{x}_{\mathrm{j}}\right) \\
& \left.-\left.\mathrm{G}\left(\mathrm{e}_{\mathrm{i}}\right)\left(\mathrm{x}_{\mathrm{j}}\right)\right|^{2}\right\}^{1 / 2}
\end{aligned}
$$

4. The normalized Euclidean distance

$$
\begin{aligned}
\mathrm{d}_{\mathrm{nE}}((\mathrm{F}, \mathrm{A}),(\mathrm{G}, \mathrm{B}))=\frac{1}{m n}\left\{\sum_{i=1^{*}}^{m} \cdot \sum_{j=1^{*}}^{n}\right. \\
\left.\left|\mathrm{F}\left(\mathrm{e}_{\mathrm{i}}\right)\left(\mathrm{x}_{\mathrm{j}}\right)-\mathrm{G}\left(\mathrm{e}_{\mathrm{i}}\right)\left(\mathrm{x}_{\mathrm{j}}\right)\right|^{2}\right\}^{1 / 2}
\end{aligned}
$$

5.

$$
d_{r}(A ; B)=\left\{\sum_{i=1}^{m} \cdot \sum_{j=1^{*}}^{n}\left|\mathrm{~F}\left(\mathrm{e}_{\mathrm{i}}\right)\left(\mathrm{x}_{\mathrm{j}}\right)-\mathrm{G}\left(\mathrm{e}_{\mathrm{i}}\right)\left(\mathrm{x}_{\mathrm{j}}\right)\right|^{\mathrm{r}}\right\}^{1 / \mathrm{r}}
$$

6. The sup distance

$$
\left.\underset{i}{d_{\infty}(A ; B)}=\sup \mid \mathrm{F}\left(\mathrm{e}_{\mathrm{i}}\right) \mathrm{x}_{\mathrm{j}}\right)-\mathrm{G}\left(\mathrm{e}_{\mathrm{i}}\right)\left(\mathrm{x}_{\mathrm{j}}\right) \mid
$$

\section{Some Similarity Measure of Fuzzy Soft Set}

In this section we define similarity measure of fuzzy soft sets based on matching function, set theoretic approach and distance.

Let $(\mathrm{F}, \mathrm{A})$ and $(\mathrm{G}, \mathrm{B})$ be two fuzzy soft sets over $(\mathrm{U}, \mathrm{E})$. Then similarity measure between $(\mathrm{F}, \mathrm{A})$ and $(\mathrm{G}, \mathrm{B})$ is defined as $\mathrm{s}((\mathrm{F}, \mathrm{A}),(\mathrm{G}, \mathrm{B}))$, which satisfies the following properties.

$$
\begin{aligned}
& \text { (S1) } 0 \leq \mathrm{s}((\mathrm{F}, \mathrm{A}),(\mathrm{G}, \mathrm{B})) \leq 1 \text {; } \\
& \text { (S2) } \mathrm{s}((\mathrm{F}, \mathrm{A}),(\mathrm{G}, \mathrm{B}))=1 \text {, if }(\mathrm{F}, \mathrm{A})=(\mathrm{G}, \mathrm{B}) \text {; } \\
& \text { (S3) } \mathrm{s}(\mathrm{F}, \mathrm{A}),(\mathrm{G}, \mathrm{B}))=\mathrm{s}((\mathrm{G}, \mathrm{B}),(\mathrm{F}, \mathrm{A})) \text {; } \\
& \text { (S4) Let }(\mathrm{H}, \mathrm{C}) \text { be a fuzzy soft set, } \\
& \quad \text { if }(\mathrm{F}, \mathrm{A}) \subseteq(\mathrm{G}, \mathrm{B}) \subseteq(\mathrm{H}, \mathrm{C}),
\end{aligned}
$$

then $\mathrm{s}((\mathrm{F}, \mathrm{A}),(\mathrm{H}, \mathrm{C})) \leq \mathrm{s}((\mathrm{F}, \mathrm{A}),(\mathrm{G}, \mathrm{B}))$ and $\mathrm{s}((\mathrm{F}, \mathrm{A}),(\mathrm{H}, \mathrm{C})) \leq$ $\mathrm{s}((\mathrm{G}, \mathrm{B}),(\mathrm{H}, \mathrm{C}))$.

Throughout this, we assume that the fuzzy soft sets $(F, A)$ and $(\mathrm{G}, \mathrm{B})$ have the same parameters.

Let $(\mathrm{F}, \mathrm{E})=\left\{\mathrm{F}\left(\mathrm{e}_{\mathrm{i}}\right) \quad, \quad \mathrm{i}=1,2,3, \ldots . \mathrm{n}\right\}$ and $(\mathrm{G}, \mathrm{E})=\left\{\mathrm{G}\left(\mathrm{e}_{\mathrm{i}}\right)\right.$, $\mathrm{i}=1,2,3, \ldots \ldots \mathrm{n}\}$ be two fuzzy soft sets where $\mathrm{F}\left(\mathrm{e}_{\mathrm{i}}\right)$ is the $\mathrm{e}_{\mathrm{i}}$ approximations of $(F, E)$ and $G\left(e_{i}\right)$ is the $e_{i}$ approximations of $(\mathrm{G}, \mathrm{E})$. Now we define a normalized Hamming distance and normalized Euclidean distance in fuzzy soft sets as follows.

\section{Volume 4 Issue 11, November 2015}




\section{International Journal of Science and Research (IJSR) \\ ISSN (Online): 2319-7064}

Index Copernicus Value (2013): 6.14 | Impact Factor (2014): 5.611

Majumdar and Samanta have defined following distance between soft set as follows

\section{Definition 4.1}

Let $U$ be an initial universal set and let $E$ be a set of parameters. Let $I^{U}$ denote the collection of all fuzzy subsets of $U$. A pair $(\mathrm{F}, \mathrm{E})$ is called a fuzzy soft set over $\mathrm{U}$, where $F$ is a mapping given by $F: E \rightarrow I^{U}$.

The similarity measures based on matching function of fuzzy soft sets as follows

Let $U$ be the universe and $E$, the set of parameters. Then we can express a fuzzy soft set over $U$. Let $(\mathrm{F}, \mathrm{E})=\left\{\mathrm{F}\left(\mathrm{e}_{\mathrm{i}}\right)\right.$, $\mathrm{i}=1,2,3, \ldots . . \mathrm{n}\}$ and $(\mathrm{G}, \mathrm{E})=\left\{\mathrm{G}\left(\mathrm{e}_{\mathrm{i}}\right), \mathrm{i}=1,2,3, \ldots . \mathrm{n}\right\}$ be two fuzzy soft sets where $F\left(e_{i}\right)$ is the $e_{i}$ approximations of $(F, E)$ and $\mathrm{G}\left(\mathrm{e}_{\mathrm{i}}\right)$ is the $e_{i}$ approximations of $(\mathrm{G}, \mathrm{E})$.

\section{Definition 4.2}

Let $(F, E)$ and $(G, E)$ be two fuzzy soft sets over $U$. Then the similarity between them, denoted by $S(F, G)$ or $S_{F, G}$ is defined by

$$
S_{F, G}=\frac{\sum_{j=1}^{n}\left\{\mathrm{~F}\left(\mathrm{e}_{\mathrm{i}}\right)\left(\mathrm{x}_{\mathrm{j}}\right) \cdot \mathrm{G}\left(\mathrm{e}_{\mathrm{i}}\right)\left(\mathrm{x}_{\mathrm{j}}\right)\right\}}{\sum_{j=1}^{n}\left\{\left(\mathrm{~F}\left(\mathrm{e}_{\mathrm{i}}\right)\left(\mathrm{x}_{\mathrm{j}}\right)\right)^{2} \cdot\left(\mathrm{G}\left(\mathrm{e}_{\mathrm{i}}\right)\left(\mathrm{x}_{\mathrm{j}}\right)\right)^{2}\right\}}
$$

The similarity measures based on set theoretic approach of fuzzy soft sets as follows.

Let $U$ be the universe and $E$, the set of parameters. Then we can express a fuzzy soft set over $U$. Let $(\mathrm{F}, \mathrm{E})=\left\{\mathrm{F}\left(\mathrm{e}_{\mathrm{i}}\right)\right.$, $\mathrm{i}=1,2,3, \ldots . \mathrm{n}\}$ and $(\mathrm{G}, \mathrm{E})=\left\{\mathrm{G}\left(\mathrm{e}_{\mathrm{i}}\right), \mathrm{i}=1,2,3, \ldots . \mathrm{n}\right\}$ be two fuzzy soft sets where $F\left(e_{i}\right)$ is the $e_{i}$ approximations of $(F, E)$ and $\mathrm{G}\left(\mathrm{e}_{\mathrm{i}}\right)$ is the $\mathrm{e}_{\mathrm{i}}$ approximations of $(\mathrm{G}, \mathrm{E})$.

\section{Definition 4.3}

Let $(F, E)$ and $(G, E)$ be two fuzzy soft sets over $U$. Then the similarity between them, denoted by $M(F, G)$ or $M_{F, G}$ is defined by

$$
M_{F, G}=\frac{\sum_{j=1^{*}}^{n}\left\{\mathrm{~F}_{\mathrm{ij}}\left(\mathrm{e}_{\mathrm{i}}\right)\left(\mathrm{x}_{\mathrm{j}}\right) \wedge \mathrm{G}_{\mathrm{ij}}\left(\mathrm{e}_{\mathrm{i}}\right)\left(\mathrm{x}_{\mathrm{j}}\right)\right.}{\sum_{j^{n}=1^{*}}^{n}\left\{\left(\mathrm{~F}_{\mathrm{ij}}\left(\mathrm{e}_{\mathrm{i}}\right)\left(\mathrm{x}_{\mathrm{j}}\right)\right) \vee\left(\mathrm{G}_{\mathrm{ij}}\left(\mathrm{e}_{\mathrm{i}}\right)\left(\mathrm{x}_{\mathrm{j}}\right)\right)\right\}}
$$

\section{Examples 4.4}

Consider the following two fuzzy soft sets where $E=\left\{\mathrm{e}_{1}, \mathrm{e}_{2}\right\}$ and $\mathrm{U}=\left\{\mathrm{x}_{1}, \mathrm{x}_{2}, \mathrm{x}_{3}, \mathrm{x}_{4}, \mathrm{x}_{5}\right\}$

Table 1.1: Fuzzy soft set (F, E)

\begin{tabular}{|c|c|c|c|c|c|}
\hline$(\mathbf{F}, \mathbf{E})$ & $\mathrm{x}_{1}$ & $\mathrm{x}_{2}$ & $\mathrm{x}_{3}$ & $\mathrm{x}_{4}$ & $\mathrm{x}_{5}$ \\
\hline $\mathbf{e}_{\mathbf{1}}$ & 0.3 & 0 & 0.7 & 0.2 & 0.2 \\
\hline $\mathbf{e}_{\mathbf{2}}$ & 0.8 & 0.2 & 0.3 & 0.5 & 0.2 \\
\hline
\end{tabular}

and

Table 1.2: Fuzzy soft set (G, E)

\begin{tabular}{|c|c|c|c|c|c|}
\hline$(\mathbf{G , E})$ & $\mathrm{x}_{1}$ & $\mathrm{x}_{2}$ & $\mathrm{x}_{3}$ & $\mathrm{x}_{4}$ & $\mathrm{x}_{5}$ \\
\hline $\mathbf{e}_{\mathbf{1}}$ & 0.1 & 0.4 & 0.5 & 0.3 & 0.3 \\
\hline $\mathbf{e}_{\mathbf{2}}$ & 0.3 & 0.4 & 0.2 & 0.1 & 0.9 \\
\hline
\end{tabular}

$$
\begin{aligned}
& \sum_{j=1 *}^{n}\left\{\mathrm{~F}_{\mathrm{i} 1}\left(\mathrm{e}_{\mathrm{i}}\right)\left(\mathrm{x}_{\mathrm{j}}\right) \wedge \mathrm{G}_{\mathrm{i} 1}\left(\mathrm{e}_{\mathrm{i}}\right)\left(\mathrm{x}_{\mathrm{j}}\right)\right\} \\
& M_{1}(F, G)= \\
& =\frac{0.1+0.3}{-----}=\frac{0.4}{0.3+0.8}=0.36 \\
& \sum_{j=1^{*}}^{n}\left\{\mathrm{~F}_{\mathrm{i} 2}\left(\mathrm{e}_{\mathrm{i}}\right)\left(\mathrm{x}_{\mathrm{j}}\right) \wedge \mathrm{G}_{\mathrm{i} 2}\left(\mathrm{e}_{\mathrm{i}}\right)\left(\mathrm{x}_{\mathrm{j}}\right)\right\} \\
& M_{2}(F, G)= \\
& =\frac{0.0+0.2}{----0.2}=\frac{0.2}{0.4+0.4}=0.25 \\
& \sum_{j=1^{*}}^{n}\left\{\mathrm{~F}_{\mathrm{i} 3}\left(\mathrm{e}_{\mathrm{i}}\right)\left(\mathrm{x}_{\mathrm{j}}\right) \wedge \mathrm{G}_{\mathrm{i} 3}\left(\mathrm{e}_{\mathrm{i}}\right)\left(\mathrm{x}_{\mathrm{j}}\right)\right\} \\
& M_{3}(F, G)= \\
& \sum_{j=1 \times}^{n}\left\{\left(\mathrm{~F}_{\mathrm{i} 3}\left(\mathrm{e}_{\mathrm{i}}\right)\left(\mathrm{x}_{\mathrm{j}}\right)\right) \vee\left(\mathrm{G}_{\mathrm{i} 3}\left(\mathrm{e}_{\mathrm{i}}\right)\left(\mathrm{x}_{\mathrm{j}}\right)\right)\right\} \\
& =\frac{0.5+0.2}{-----0.7}=\frac{----}{0.7+0.3}=0.7 \\
& \sum_{j=1^{*}}^{n}\left\{\mathrm{~F}_{\mathrm{i} 4}\left(\mathrm{e}_{\mathrm{i}}\right)\left(\mathrm{x}_{\mathrm{j}}\right) \wedge \mathrm{G}_{\mathrm{i} 4}\left(\mathrm{e}_{\mathrm{i}}\right)\left(\mathrm{x}_{\mathrm{j}}\right)\right.
\end{aligned}
$$

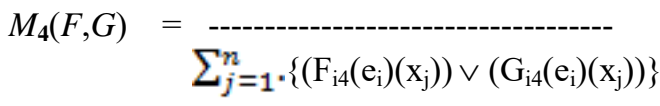

$$
\begin{aligned}
& =\frac{0.2+0.1}{-----0.3}=\frac{0.3}{----}=0.37 \\
& \sum_{j=1^{*}}^{n}\left\{\mathrm{~F}_{\mathrm{i} 5}\left(\mathrm{e}_{\mathrm{i}}\right)\left(\mathrm{x}_{\mathrm{j}}\right) \wedge \mathrm{G}_{\mathrm{i} 5}\left(\mathrm{e}_{\mathrm{i}}\right)\left(\mathrm{x}_{\mathrm{j}}\right)\right\} \\
& M_{5}(F, G)=\frac{\sum_{j=1}^{*}}{\sum^{n}}\left\{\left(\mathrm{~F}_{\mathrm{i} 5}\left(\mathrm{e}_{\mathrm{i}}\right)\left(\mathrm{x}_{\mathrm{j}}\right)\right) \vee\left(\mathrm{G}_{\mathrm{i} 5}\left(\mathrm{e}_{\mathrm{i}}\right)\left(\mathrm{x}_{\mathrm{j}}\right)\right)\right\} \\
& =\frac{0.2+0.2}{-------}=\frac{0.4}{0.3+0.9}=0.33
\end{aligned}
$$

Thus we have

$$
\begin{aligned}
M(F, G)=\operatorname{Max} & \left\{M_{\mathrm{j}}(F, G)\right\}, \mathrm{j}=1,2,3,4,5 . \\
= & \operatorname{Max}\left\{\mathrm{M}_{1}, \mathrm{M}_{2}, \mathrm{M}_{3}, \mathrm{M}_{4}, \mathrm{M}_{5}\right\} \\
& =\mathrm{M}_{3} \\
& =0.7
\end{aligned}
$$

\section{Application of Similarity Measure of Fuzzy Soft Set based in Decision Making:}

Let $U=\left\{x_{1}, x_{2} \ldots\right\}$ be the universal set of elements and $\mathrm{E}=\left\{\mathrm{e}_{1}, \mathrm{e}_{2} \ldots\right\}$ be the universal set of parameters.

Let $F=(F, E)$ and $G=(G, E)$ be two fuzzy soft sets over $(\mathrm{U}, \mathrm{E})$, then $(\mathrm{F}, \mathrm{E})=\left\{\mathrm{F}\left(\mathrm{e}_{\mathrm{i}}\right) \in \mathrm{P}(\mathrm{U}), \mathrm{i}=1,2,3, \ldots . \mathrm{n}\right\}$ and $(\mathrm{G}, \mathrm{E})=$ $\left\{\mathrm{G}\left(\mathrm{e}_{\mathrm{i}}\right) \in \mathrm{P}(\mathrm{U}), \mathrm{i}=1,2,3, \ldots . \mathrm{n}\right\}$ where $\mathrm{F}\left(\mathrm{e}_{\mathrm{i}}\right)$ is the $\mathrm{e}_{\mathrm{i}}$ 


\section{International Journal of Science and Research (IJSR) \\ ISSN (Online): 2319-7064}

Index Copernicus Value (2013): 6.14 | Impact Factor (2014): 5.611

approximations of $(F, E)$ and $G\left(e_{i}\right)$ is the $e_{i}$ approximations we have

of $(\mathrm{G}, \mathrm{E}), \mathrm{P}(\mathrm{U})$ be the collection of all fuzzy subsets of $\mathrm{U}$.

Let we define

$$
\sum_{j=1 \times}^{n}\left\{\mathrm{~F}_{\mathrm{ij}}\left(\mathrm{e}_{\mathrm{i}}\right)\left(\mathrm{x}_{\mathrm{j}}\right) \wedge \mathrm{G}_{\mathrm{ij}}\left(\mathrm{e}_{\mathrm{i}}\right)\left(\mathrm{x}_{\mathrm{j}}\right)\right\}
$$

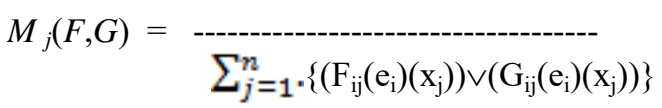

Where $F_{i j}=F\left(e_{i}\right)\left(x_{i}\right) \in I$ and $G_{i j}=G\left(e_{i}\right)\left(x_{i}\right) \in I$ then

$$
\mathrm{M}_{\mathrm{F}, \mathrm{G}}=\operatorname{Max}\left\{\mathrm{M}_{\mathrm{j}}(\mathrm{F}, \mathrm{G})\right\}, \mathrm{j}=1,2, \ldots \mathrm{n} \text {. }
$$

Analogous and majumder and samanta [7], we would call the fuzzy soft sets $(F, E)$ and $(G, E)$ in the fuzzy soft class $(U, E)$ to be significantly . Similar if $M((F, E),(G, E))>1 / 2$

Suppose that $U=\left\{S_{1}, S_{2}, S_{3}, S_{4}\right\}$ be the set of four subjects under consideration and $\mathrm{E}=\left\{\mathrm{e}_{1}, \mathrm{e}_{2}, \mathrm{e}_{3}, \mathrm{e}_{4}\right\}$ is the set of parameters indicating specific for the students

$$
\begin{aligned}
& \mathrm{e}_{1}=\text { Gifted Children } \\
& \mathrm{e}_{2}=\text { Specially Talented Children } \\
& \mathrm{e}_{3}=\text { Average learner } \\
& \mathrm{e}_{4}=\text { slow learner }
\end{aligned}
$$

$$
\begin{aligned}
& \sum_{j=1^{*}}^{n}\left\{\mathrm{~F}_{\mathrm{i} 1}\left(\mathrm{e}_{\mathrm{i}}\right)\left(\mathrm{x}_{\mathrm{j}}\right) \wedge \mathrm{G}_{\mathrm{i} 1}\left(\mathrm{e}_{\mathrm{i}}\right)\left(\mathrm{x}_{\mathrm{j}}\right)\right\} \\
& M_{1}(F, G)=\frac{\sum_{j=1}^{n}}{\sum_{j}}\left\{\left(\mathrm{~F}_{\mathrm{i} 1}\left(\mathrm{e}_{\mathrm{i}}\right)\left(\mathrm{x}_{\mathrm{j}}\right)\right) \vee\left(\mathrm{G}_{\mathrm{i} 1}\left(\mathrm{e}_{\mathrm{i}}\right)\left(\mathrm{x}_{\mathrm{j}}\right)\right)\right\} \\
& M_{1}(F, G)=\frac{0.2+0.7+0.2+0.1}{0.9+0.8+0.8+0.8}=\frac{1.2}{---}=0.36 \\
& M_{2}(F, G)=\frac{0.3+0.6+0.1+0.2}{0.6+0.7+0.5+0.8}=\begin{array}{c}
1.2 \\
----
\end{array}=0.4 \\
& M_{3}(F, G) \quad=\frac{0.4+0.4+0.2+0.2}{0.8+0.6+0.8+0.3}=\frac{1.2}{2.5}=0.48 \\
& M_{4}(F, G)=\frac{0.7+0.2+0.3+0.2}{0.9+0.4+0.9+0.8}=\frac{1.4}{----}=0.46 \\
& M_{F, G}=\operatorname{Max}\left\{M_{j}(F, G)\right\}, j=1,2, \ldots n . \\
& M_{F, G}=\operatorname{Max}\{0.36,0.4,0.48,0.46\}=0.48<1 / 2
\end{aligned}
$$

Fuzzy soft sets for the performing students $(F, E)$ is given in table 1.Similarly we construct the fuzzy soft sets for the three students under consideration as given .

Table 1: Fuzzy Soft Set For Performing Students.

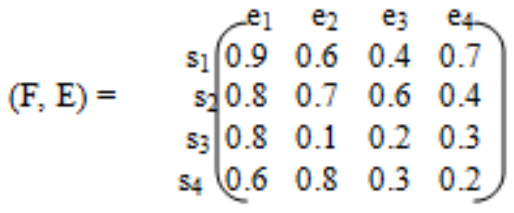

Table 2: Fuzzy Soft Set for First Students under consideration

$$
(\mathrm{G}, \mathrm{E})=\mathrm{s}_{1}\left(\begin{array}{cccc}
e_{1} & e_{2} & e_{3} & e_{4} \\
\mathrm{~s}_{2} & 0.3 & 0.8 & 0.9 \\
\mathrm{~s}_{3} & \mathrm{~s}_{4}
\end{array}\left(\begin{array}{llll}
0.7 & 0.6 & 0.4 & 0.2 \\
0.2 & 0.5 & 0.8 & 0.9 \\
0.1 & 0.2 & 0.2 & 0.8
\end{array}\right)\right.
$$

Table 3: Fuzzy Soft Set for Second Students under consideration

$$
(H, E)=s_{1}\left(\begin{array}{cccc}
e_{1} & e_{2} & e_{3} & e_{4} \\
0.4 & 0.2 & 0.5 & 0.8 \\
0.9 & 0.8 & 0.7 & 0.6 \\
s_{3} & s_{4}
\end{array}\left(\begin{array}{llll}
0.4 & 0.3 & 0.1 & 0.2 \\
0.3 & 0.5 & 0.8 & 0.1
\end{array}\right)\right.
$$

Table 4: Fuzzy Soft Set For Third Students under consideration

$$
(I, E)=s_{1}\left(\begin{array}{cccc}
e_{1} & e_{2} & e_{3} & e_{4} \\
s_{2} & 0.4 & 0.8 & 0.4 \\
0.7 & 0.9 & 0.5 & 0.2 \\
s_{3} & s_{4}
\end{array}\left(\begin{array}{cccc}
0.2 & 0.6 & 0.8 & 0.1 \\
0.1 & 0.2 & 0.1 & 0.5
\end{array}\right)\right.
$$

Similarly

$M_{1}(F, H)=0.55, M_{\mathbf{2}}(F, H)=0.60, M_{3}(F, H)=0.63, M_{\mathbf{4}}(F, H)=$ 0.73

$M_{F, H}=\operatorname{Max}\{0.55,0.60,0.63,0.73\}=0.73>1 / 2$

and

$M_{1}(F, I)=0.48, M_{\mathbf{2}}(F, I)=0.48, \quad M_{\mathbf{3}}(F, I)=0.48, M_{\mathbf{4}}(F, I)=$ 0.47

$M_{\text {F,I }}=\operatorname{Max}\{0.48,0.48,0.48,0.47\}=0.48<1 / 2$

\section{Evaluate Results}

\begin{tabular}{|c|c|c|c|}
\hline Similarity & $\begin{array}{c}\text { First } \\
\text { student }\end{array}$ & $\begin{array}{c}\text { Second } \\
\text { student }\end{array}$ & $\begin{array}{c}\text { Third } \\
\text { student }\end{array}$ \\
\cline { 2 - 4 } & 0.48 & 0.73 & 0.48 \\
\hline
\end{tabular}

Therefore, The Result is Second Student may be best student.

Next, we proceed similarity measures based on the normalized Hamming distance and normalized Euclidean distance measure of fuzzy soft sets as follows.

\section{Definition 5.1}

Suppose (F,A) and (G,B) be two fuzzy soft sets over U, the similarity measure based on the normalized Hamming distance between them is defined as

$$
\begin{array}{r}
\mathrm{S}_{\mathrm{nH}}((\mathrm{F}, \mathrm{A}),(\mathrm{G}, \mathrm{B}))=1-\frac{1}{m n} \sum_{i=1}^{m} \sum_{j=1 *}^{n} \mid \mathrm{F}\left(\mathrm{e}_{\mathrm{i}}\right)\left(\mathrm{x}_{\mathrm{j}}\right) \\
-\mathrm{G}\left(\mathrm{e}_{\mathrm{i}}\right)\left(\mathrm{x}_{\mathrm{j}}\right) \mid
\end{array}
$$




\section{International Journal of Science and Research (IJSR) \\ ISSN (Online): 2319-7064}

Index Copernicus Value (2013): 6.14 | Impact Factor (2014): 5.611

Definition 5.2

Suppose $(F, A)$ and $(G, B)$ be two fuzzy soft sets over $U$, the similarity measure based on the normalized Hamming distance between them is defined as

$$
\begin{array}{r}
\mathrm{S}_{\mathrm{nE}}((\mathrm{F}, \mathrm{A}),(\mathrm{G}, \mathrm{B}))=1-\frac{1}{m n}\left\{\sum_{\mathrm{i}=1^{*}}^{m} \sum_{j=1}^{n} \mid \mathrm{F}\left(\mathrm{e}_{\mathrm{i}}\right)\left(\mathrm{x}_{\mathrm{j}}\right)\right. \\
\left.-\left.\mathrm{G}\left(\mathrm{e}_{\mathrm{i}}\right)\left(\mathrm{x}_{\mathrm{j}}\right)\right|^{2}\right\}^{1 / 2}
\end{array}
$$

\section{Application of Similarity Measure of Fuzzy Soft Set based on distance in Medical Diagnosis}

Suppose that there are three patients G, H, I in a hospital with symptoms high fever, Fatigue vomiting, muscle pain, cough and Skin rash problem. Let the universal set contain only three elements ,high ${ }^{\text {ee }}(\mathrm{h})$, "medium $(\mathrm{m})$ and ,normal ${ }^{\text {ee }}(\mathrm{n})$. ie., $U=\{h, m, n\}$. Here the set of parameters $E$ is the set of certain approximations determined by the Hospital.

Let $\mathrm{E}=\left\{\mathrm{e}_{1} \mathrm{e}_{2}, \mathrm{e}_{3}, \mathrm{e}_{4}, \mathrm{e}_{5}, \mathrm{e}_{6\}}\right.$, where $\mathrm{e}_{1}=$ high fever, $\mathrm{e}_{2}=$ Fatigue, $\mathrm{e}_{3}=$ vomiting, $\mathrm{e}_{4}=$ joint pain, $\mathrm{e}_{5}=$ cough, $\mathrm{e}_{6}=$ Skin rash.

We construct the fuzzy soft set $(F, E)$ for dengue from medical knowledge as given in Table1.1

Table 1.1: Fuzzy soft set (F, E) for fever

\begin{tabular}{|c|c|c|c|c|c|}
\hline $\mathbf{F , E )}$ & $\mathbf{e}_{\mathbf{1}}$ & $\mathbf{e}_{\mathbf{2}}$ & $\mathbf{e}_{\mathbf{3}}$ & $\mathbf{e}_{\mathbf{4}}$ & $\mathbf{e}_{\mathbf{5}}$ \\
\hline $\mathbf{H}$ & 1 & 1 & 0.4 & 1 & 0 \\
\hline $\mathbf{M}$ & 0.7 & 0.6 & 0.4 & 0.5 & 0.4 \\
\hline $\mathbf{N}$ & 0.3 & 0.1 & 0.6 & 0 & 1 \\
\hline
\end{tabular}

Similarly, we construct the fuzzy soft sets for the three patients under consideration as given in Table 1.2, 1.3 and 1.4

Table 1.2: Fuzzy soft set $(\mathrm{G}, \mathrm{E})$ for the first patient.

\begin{tabular}{|c|c|c|c|c|c|}
\hline$(\mathbf{G}, \mathbf{E})$ & $\mathbf{e}_{\mathbf{1}}$ & $\mathbf{e}_{\mathbf{2}}$ & $\mathbf{e}_{\mathbf{3}}$ & $\mathbf{e}_{\mathbf{4}}$ & $\mathbf{e}_{\mathbf{5}}$ \\
\hline $\mathbf{H}$ & 0.1 & 0.8 & 0.3 & 0.8 & 0 \\
\hline $\mathbf{M}$ & 0.2 & 0.2 & 0.7 & 0.1 & 0.2 \\
\hline $\mathbf{N}$ & 1 & 0.1 & 0.2 & 0 & 0.5 \\
\hline
\end{tabular}

Table 1.3: Fuzzy soft set (H,E) for the second patient

\begin{tabular}{|c|c|c|c|c|c|}
\hline$(\mathbf{H}, \mathbf{E})$ & $\mathbf{e}_{\mathbf{1}}$ & $\mathbf{e}_{\mathbf{2}}$ & $\mathbf{e}_{\mathbf{3}}$ & $\mathbf{e}_{\mathbf{4}}$ & $\mathbf{e}_{\mathbf{5}}$ \\
\hline $\mathbf{H}$ & 0.6 & 0.8 & 0.3 & 0.6 & 0.4 \\
\hline $\mathbf{M}$ & 0.3 & 0.2 & 0.2 & 0.1 & 0.1 \\
\hline $\mathbf{N}$ & 0.1 & 0.1 & 0.5 & 0.3 & 0.6 \\
\hline
\end{tabular}

Table 1.4: Fuzzy soft set (I,E) for the third patient

\begin{tabular}{|c|c|c|c|c|c|}
\hline$(\mathbf{I}, \mathbf{E})$ & $\mathbf{e}_{\mathbf{1}}$ & $\mathbf{e}_{\mathbf{2}}$ & $\mathbf{e}_{\mathbf{3}}$ & $\mathbf{e}_{\mathbf{4}}$ & $\mathbf{e}_{\mathbf{5}}$ \\
\hline $\mathbf{H}$ & 0.2 & 0.1 & 0.5 & 0.3 & 0.5 \\
\hline $\mathbf{M}$ & 0.5 & 0.2 & 0.4 & 0.7 & 0.3 \\
\hline $\mathbf{N}$ & 0.6 & 0.6 & 0.2 & 0.2 & 0.2 \\
\hline
\end{tabular}

We calculate the normalized Hamming distance between $(\mathrm{F}, \mathrm{E})$ and $(\mathrm{G}, \mathrm{E})$.

$$
\begin{aligned}
& \mathrm{d}_{\mathrm{nH}}((\mathrm{F}, \mathrm{E}),(\mathrm{G}, \mathrm{E}))=\frac{1}{m n} \sum_{\mathrm{i}=1^{*}}^{m} \sum_{j=1^{*}}^{n} \mid \mathrm{F}\left(\mathrm{e}_{\mathrm{i}}\right)\left(\mathrm{x}_{\mathrm{j}}\right)-\mathrm{G}\left(\mathrm{e}_{\mathrm{i}}\right)\left(\mathrm{x}_{\mathrm{j}}\right) \\
& =\frac{1}{3 x^{6}}[(0.9+0.5+0.7)+(0.2+0.4+0)+(0.1+0.3+0.4)+
\end{aligned}
$$

$$
\begin{aligned}
& =\frac{1}{3 x^{6}}[2.1+0.6+0.8+0.6+0.7+0.7] \\
& =0.31 \\
& \text { Similarly, } \\
& \mathbf{d}_{\mathbf{n H}}((\mathbf{F}, \mathbf{E}),(\mathbf{H}, \mathbf{E}))=\mathbf{0 . 3} ; \mathbf{d}_{\mathbf{n H}}((\mathbf{F}, \mathbf{E}),(\mathbf{I}, \mathbf{E}))=\mathbf{0 . 4 3 3}
\end{aligned}
$$

Then, We calculate the normalized Euclidean distance between $(F, E)$ and $(\mathrm{G}, \mathrm{E})$.

$$
\begin{aligned}
& \mathbf{d}_{\mathbf{n E}}((\mathbf{F}, \mathbf{E}),(\mathbf{G}, \mathbf{E}))=\frac{1}{m n}\left\{\sum_{i=1}^{m} \cdot \sum_{j=1}^{n} \times\left|F\left(\mathrm{e}_{\mathrm{i}}\right)\left(\mathrm{x}_{\mathrm{j}}\right)-\mathrm{G}\left(\mathrm{e}_{\mathrm{i}}\right)\left(\mathrm{x}_{\mathrm{j}}\right)\right|^{2}\right\}^{1 / 2} \\
& =\frac{1}{3 x 6}\left[2.1^{2}+0.6^{2}+0.8^{2}+0.6^{2}+0.7^{2}+0.7^{2}\right] \\
& =\mathbf{0 . 3 7 5}
\end{aligned}
$$

Similarly

$\mathrm{d}_{\mathrm{nE}}((\mathrm{F}, \mathrm{E}),(\mathrm{H}, \mathrm{E}))=0.29 ; \quad \mathrm{d}_{\mathrm{nE}}((\mathrm{F}, \mathrm{E}),(\mathrm{I}, \mathrm{E}))=0.62$

\section{Similarity Measure on normalized Hamming distance:}

$$
\begin{aligned}
& \mathrm{S}_{\mathrm{nH}}((\mathrm{F}, \mathrm{E}),(\mathrm{G}, \mathrm{E}))=1-\mathrm{d}_{\mathrm{nH}}((\mathrm{F}, \mathrm{E}),(\mathrm{G}, \mathrm{E}))=1-0.31=0.694 \\
& \mathrm{~S}_{\mathrm{nH}}((\mathrm{F}, \mathrm{E}),(\mathrm{H}, \mathrm{E}))=1-\mathrm{d}_{\mathrm{nH}}((\mathrm{F}, \mathrm{E}),(\mathrm{H}, \mathrm{E})=1-0.30=0.70 \\
& \mathrm{S}_{\mathrm{nH}}((\mathrm{F}, \mathrm{E}),(\mathrm{I}, \mathrm{E}))=1-\mathrm{d}_{\mathrm{nH}}((\mathrm{F}, \mathrm{E}),(\mathrm{I}, \mathrm{E}))=1-0.43=0.567
\end{aligned}
$$

\section{Similarity Measure on normalized Euclidean distance:}

$$
\begin{aligned}
& \mathrm{S}_{\mathrm{nE}}((\mathrm{F}, \mathrm{E}),(\mathrm{G}, \mathrm{E}))=1-\mathrm{d}_{\mathrm{nE}}((\mathrm{F}, \mathrm{E}),(\mathrm{G}, \mathrm{E}))=1-0.375=0.625 \\
& \mathrm{~S}_{\mathrm{nE}}((\mathrm{F}, \mathrm{E}),(\mathrm{H}, \mathrm{E}))=1-\mathrm{d}_{\mathrm{nE}}((\mathrm{F}, \mathrm{E}),(\mathrm{H}, \mathrm{E}))=1-0.29=0.70 \\
& \mathrm{~S}_{\mathrm{nE}}((\mathrm{F}, \mathrm{E}),(\mathrm{I}, \mathrm{E}))=1-\mathrm{d}_{\mathrm{nE}}((\mathrm{F}, \mathrm{E}),(\mathrm{I}, \mathrm{E}))=1-0.62=0.37
\end{aligned}
$$

Table 1.5: Evaluate Results

\begin{tabular}{|c|c|c|c|}
\hline Similarity & $\begin{array}{c}\text { First } \\
\text { patient }\end{array}$ & $\begin{array}{l}\text { Second } \\
\text { patient }\end{array}$ & $\begin{array}{c}\text { Third } \\
\text { patient }\end{array}$ \\
\hline Normalized Hamming Distance & 0.694 & 0.7 & 0.567 \\
\hline Normalized Euclidean Distance & 0.625 & 0.7 & 0.37 \\
\hline
\end{tabular}

Therefore, the result is (second patient $>$ the first patient $>$ the third patient)

\section{Conclusion}

In view of our discussion, We have applied the new similarity of fuzzy soft sets in decision making problem based on set theoretic approach and medical diagnosis based on distance measure. First we can conclude that decision making problem based on set theoretic approach, Second Student may be best student and medical diagnosis based on distance measure from Table 1.5 , second patient is suffering from dengue. It is hoped that our work would help enhancing this study on fuzzy soft sets

\section{References}

[1] B.Ahmad and Athar Kharal,"On Fuzzy Soft Sets", Advances in Fuzzy Systems,2009. 
[2] N.Cagman, S.Enginoglu and F.Citak, Fuzzy soft set theory and its application, Iran. J. Fuzzy Syst., 8 (2011) 137-147

[3] Dusmanta Kumar Sut, "An Application of Similarity of Fuzzy Soft sets in Decision Making" Int.J.Computer Technology \& Applications, Vol 3(2), 742-745

[4] Q.Feng, W.Zheng, "New Similarity Measures of Fuzzy Soft Sets Based on Distance Measures, annals of fuzzy mathematics and information, Sep 2013

[5] A. Kharal, "Distance and Similarity measures for soft sets", New Math. Nat. Comput., 6 (2010) ,321-334.

[6] P.K.Maji, R.Biswas and A.R.Roy, "Fuzzy Soft Sets", Journal of Fuzzy Mathematics,9(3), 2001, 589-602.

[7] P.Majumder and S.K. Samantha,"On Similarity Measure of Fuzzy Soft Sets", Int.J.Advance Soft Comput.Appl., vol.3, No.2, July 2011.

[8] D.A.Molodstov,"Soft set theory-first result", Computers and Mathematics with Applications, 37, 1999, 19-31.

[9] Dr. P. Rajarajeswari and P. Dhanalakshmi, "An application of similarity measure of Fuzzy soft set based on distance", Journal of Mathematics, 4 (2012) 27-30.

[10] Sarala and Suganya, "Some Properties of Fuzzy Soft Groups" . IOSR Journal of Mathematics (IOSR-JM) Volume 10, Issue 2 Ver. III (Mar-Apr.2014), P 36-40,

[11] Sarala and Suganya," On Normal Fuzzy Soft Groups". International Journal of Mathematics Trend and Technology - Volume 10 Number 2 - Jun 2014,

[12] Sarala and Suganya," Q- Fuzzy Soft Ring", International Journal of Engineering Research \& Technology (IJERT), Vol. 4 Issue 02, February-2015

[13] Sarala and Suganya, "The Maximum and Minimum Conditions in Fuzzy Soft Ideals of a Fuzzy Soft Lattice", International Journal of Science and Research (IJSR), Volume 4 Issue 4, April 2015, pp:1467-1470 